\title{
Change detection in single- and multi-look polarimetric SAR data
}

Nielsen, Allan A.; Connetable, Paul J.; Conradsen, Knut; Skriver, Henning; Krogager, Ernst

Published in:

Proceedings of IEEE Radar Conference 2020

Link to article, DOI:

10.1109/RadarConf2043947.2020.9266610

Publication date:

2020

Document Version

Peer reviewed version

Link back to DTU Orbit

Citation (APA):

Nielsen, A. A., Connetable, P. J., Conradsen, K., Skriver, H., \& Krogager, E. (2020). Change detection in singleand multi-look polarimetric SAR data. In Proceedings of IEEE Radar Conference 2020 IEEE.

https://doi.org/10.1109/RadarConf2043947.2020.9266610

\section{General rights}

Copyright and moral rights for the publications made accessible in the public portal are retained by the authors and/or other copyright owners and it is a condition of accessing publications that users recognise and abide by the legal requirements associated with these rights.

- Users may download and print one copy of any publication from the public portal for the purpose of private study or research.

- You may not further distribute the material or use it for any profit-making activity or commercial gain

- You may freely distribute the URL identifying the publication in the public portal 


\title{
CHANGE DETECTION IN SINGLE- AND MULTI-LOOK POLARIMETRIC SAR DATA
}

\author{
Allan A. Nielsen ${ }^{1}$, Paul J. Connetable ${ }^{2}$, Knut Conradsen ${ }^{1}$, Henning Skriver ${ }^{2}$ and Ernst Krogager ${ }^{3}$ \\ ${ }^{1}$ Applied Mathematics and Computer Science, Technical University of Denmark \\ ${ }^{2}$ National Space Institute, Technical University of Denmark \\ ${ }^{3}$ Danish Ministry of Defence Acquisition and Logistics Organisation
}

\begin{abstract}
We describe the use of P-band data from DLR's F-SAR system to change detection between three time points. Change detection on data in the covariance matrix representation is performed by means of test statistics in the complex Wishart distribution on both multi- and single-look data. We successfully detect objects including humans buried under snow in Disko Island, Greenland.
\end{abstract}

\section{INTRODUCTION}

In the preparation to meet future challenges in the Arctic region, the Danish Defence Acquisition and Logistics Organization (DALO) has carried out several test campaigns with the F-SAR system of the German Aerospace Center (DLR). The F-SAR offers the possibility of exploring the performance of a high resolution, fully polarimetric SAR system with five frequency bands in the range of $400 \mathrm{MHz}$ to $10 \mathrm{GHz}$ [1]. Here we report on use of P-band $(435 \mathrm{MHz})$ data to change detection over three time points by means of an omnibus test statistic in the complex Wishart distribution [2]. Other results from this and other campaigns have previously been reported in [3].

Change detection is a very important method for many applications of remotely sensed data from space- and air-borne instruments. Especially SAR data are useful due to their all-weather capabilities, and hence planned acquisitions for change detection are normally secured. A number of studies have applied SAR data to change detection applications in single-channel SAR images applying different methods using e.g. the classical ratio detection $[4,5]$, the Kittler-Illingworth threshold selection criterion [6-9], hidden Markov chains for thresholding [10], wavelets [11-14], linear features [15], Kullback-Leibler divergence [16], multivariate gamma distributions [17], neural networks [18], fusion of multi-similarity measures [19], and Markov random fields [20], where most of the methods are based on the classic ratio detector and improvements thereof. Also, methods for change detection using multichannel SAR data (e.g. polarimetric) have been studied using e.g. polarimetric parameters [21,22], Markov random fields for multichannel SAR data [23, 24], generalized maximum likelihood test for covariance matrices [25] and the same test statistics for classification [26], partial vectors for suppression of the backscatter coefficient influence [27], the Hotelling-Lawley trace statistic [28], and a non-Wishart change detector [29], where a characteristic of the multichannel change detectors is that they are used for change detection between bi-temporal acquisitions.

When comparing several images one may apply the simple approach making pairwise comparisons. However, this approach makes it virtually impossible to control the rates of false positives (postulating a change when there actually is none) and of false negatives (missing an actual change). In general, a better approach for comparing several distributions is to perform a simultaneous test of the hypothesis of homogeneity of the said distributions, a so-called omnibus test, see for example [30]. Here we enable this by deriving the likelihood ratio test statistic for equality of $k \geq 2$ complex variancecovariance matrices and finding an approximation for the distribution of this test statistic under the hypothesis of equality. If the conclusion of such an analysis is that the parameters in the underlying Wishart distributions are not constant, i.e., we have a non-stationary time series, then the question naturally arises, when do the changes actually occur? Here we describe a factorization of the likelihood ratio statistic into a product of test statistics that each test simpler hypotheses of homogeneity up to a certain point and that are independent if the hypothesis of total homogeneity is true. We show how this may be used in setting up a change detector for solving the said problem.

The methods described here were first presented in [2]. Further descriptions of visualization and software in this context are given $[31,32]$.

\section{POLARIMETRIC SAR}

A fully polarimetric SAR measures the 2 by 2 complex so-called scattering matrix at each resolution cell on the ground. The scattering matrix relates the incident and the scattered electric fields, [33]. If $S_{r t}$ denotes the complex scattering amplitude for receive and transmit polarization $(r, t \in\{h, v\}$ for horizontal and vertical polarization), then reciprocity, which normally applies to natural targets, gives $S_{h v}=S_{v h}$ (in the backscattering direction using the backscattering alignment convention, [33]) and $S_{h v}$ is replaced by $\left(S_{h v}+S_{v h}\right) / \sqrt{2}$. The scattering matrix is often represented by the three-component complex target vector $\boldsymbol{s}=\left[\begin{array}{lll}S_{h h} & S_{h v} & S_{v v}\end{array}\right]^{T}$ where the superscript ${ }^{T}$ denotes the matrix transpose.

The inherent speckle in the SAR data can be reduced by spatial averaging at the expense of spatial resolution. In this so-called multilook case (below $n$ is the number of looks) a more appropriate representation of the backscattered signal is the covariance matrix in which the average properties of a group of resolution cells can be expressed in a single matrix formed by the outer products of the averaged target vectors. The sample covariance matrix is defined as [33]

$$
\begin{aligned}
\langle\boldsymbol{C}\rangle= & \left\langle\boldsymbol{s}(i) \boldsymbol{s}(i)^{H}\right\rangle \\
= & {\left[\begin{array}{lll}
\left\langle S_{h h} S_{h h}^{*}\right\rangle & \left\langle S_{h h} S_{h v}^{*}\right\rangle & \left\langle S_{h h} S_{v v}^{*}\right\rangle \\
\left\langle S_{h v} S_{h h}^{*}\right\rangle & \left\langle S_{h v} S_{h v}^{*}\right\rangle & \left\langle S_{h v} S_{v v}^{*}\right\rangle \\
\left\langle S_{v v} S_{h h}^{*}\right\rangle & \left\langle S_{v v} S_{h v}^{*}\right\rangle & \left\langle S_{v v} S_{v v}^{*}\right\rangle
\end{array}\right] }
\end{aligned}
$$

where $\langle\cdot\rangle$ denotes ensemble averaging, the ${ }^{*}$ denotes complex conjugation, and the superscript ${ }^{H}$ denotes the complex conjugate transpose. Reciprocity results in a covariance matrix with rank 3. $n\langle\boldsymbol{C}\rangle$ follows a complex Wishart distribution. 


\section{TEST STATISTICS AND THEIR DISTRIBUTIONS}

The two subsections below briefly recapitulate the main results from [2] on the test statistics $Q$ and $R_{j}$, and the corresponding probabilities $P$ (probability of change) for finding smaller values of $-2 \rho \ln Q$ and $-2 \rho_{j} \ln R_{j}$ relating to change detection in a series of $k \geq 2$ covariance matrix representation multi-look SAR data with all (including the off-diagonal) elements in the covariance matrix, see [33].

The expressions for the test statistics and the corresponding probabilities are valid also for block-diagonal covariance data, e.g., diagonal-only data which works with single-look data also, but the values of the number of degrees of freedom and parameters $\rho, \omega_{2}, \rho_{j}$, and $\omega_{2 j}$ change as given in [34].

\subsection{Test for Equality of Several Complex Covariance Matrices}

For the logarithm of the likelihood ratio test statistic $Q$ for testing whether a series of $k \geq 2$ complex variance-covariance matrices $\boldsymbol{\Sigma}_{i}$ are equal, i.e., $\boldsymbol{\Sigma}_{1}=\boldsymbol{\Sigma}_{2}=\cdots=\boldsymbol{\Sigma}_{k}$ we get (for the real case see [35]; for the case with two complex matrices see [25,36])

$$
\ln Q=n\left\{p k \ln k+\sum_{i=1}^{k} \ln \left|\boldsymbol{X}_{i}\right|-k \ln \left|\sum_{i=1}^{k} \boldsymbol{X}_{i}\right|\right\}
$$

Here $|\cdot|$ denotes the determinant, $n$ is the equivalent number of looks, the $\boldsymbol{X}_{i}$ (and the $\boldsymbol{\Sigma}_{i}$ ) are $p$ by $p(p=3$ for full pol data, $p=2$ for dual pol data, and $p=1$ for single channel power data), and the $\boldsymbol{X}_{i}=n \hat{\boldsymbol{\Sigma}}_{i}=n\langle\boldsymbol{C}\rangle_{i}$ follow the complex Wishart distribution, i.e., $\boldsymbol{X}_{i} \sim W_{C}\left(p, n, \boldsymbol{\Sigma}_{i}\right)$.

Because $Q$ tests for equality in all matrices simultaneously, it is termed an omnibus test statistic. $Q=1$ for equality.

For full and dual pol data ( $p=3$ and $p=2$ ) with all (including off-diagonal) elements in the covariance matrix and with

$$
\begin{aligned}
f & =(k-1) p^{2} \\
\rho & =1-\frac{p\left(2 p^{2}-1\right)}{6 f}\left(\frac{k}{n}-\frac{1}{n k}\right) \\
\omega_{2} & =-\frac{f}{4}\left(1-\frac{1}{\rho}\right)^{2}+\frac{p^{2}\left(p^{2}-1\right)}{24 \rho^{2}}\left(\frac{k}{n^{2}}-\frac{1}{n^{2} k^{2}}\right)
\end{aligned}
$$

the probability of finding a smaller value of $-2 \rho \ln Q$ is

$$
\begin{aligned}
& P\{-2 \rho \ln Q \leq z\} \simeq P\left\{\chi^{2}(f) \leq z\right\}+ \\
& \omega_{2}\left[P\left\{\chi^{2}(f+4) \leq z\right\}-P\left\{\chi^{2}(f) \leq z\right\}\right],
\end{aligned}
$$

where $z=-2 \rho \ln q$ and $q$ is a particular realization (the observed value) of the stochastic variable $Q$.

\subsection{Test for Equality of First $j \leq k$ Matrices}

If the above $\ln Q$ test statistic shows that we cannot reject the hypothesis $\boldsymbol{\Sigma}_{1}=\boldsymbol{\Sigma}_{2}=\cdots=\boldsymbol{\Sigma}_{k}$, there is no change over the time span covered by the data. If we can reject the hypothesis, there is change at some time point. To find that time point, we test whether the first $j(1<j \leq k)$ complex variance-covariance matrices $\boldsymbol{\Sigma}_{i}$ are equal, i.e., given that $\boldsymbol{\Sigma}_{1}=\boldsymbol{\Sigma}_{2}=\cdots=\boldsymbol{\Sigma}_{j-1}$, then the logarithm of the likelihood ratio test statistic $R_{j}$ for testing $\boldsymbol{\Sigma}_{j}=\boldsymbol{\Sigma}_{1}$ is

$$
\begin{aligned}
\ln R_{j}= & n\{p(j \ln j-(j-1) \ln (j-1))+ \\
& \left.(j-1) \ln \left|\sum_{i=1}^{j-1} \boldsymbol{X}_{i}\right|+\ln \left|\boldsymbol{X}_{j}\right|-j \ln \left|\sum_{i=1}^{j} \boldsymbol{X}_{i}\right|\right\} .
\end{aligned}
$$

Furthermore, the $R_{j}$ constitute a factorization of $Q, Q=\prod_{j=2}^{k} R_{j}$ or $\ln Q=\sum_{j=2}^{k} \ln R_{j}$.

For full and dual pol data ( $p=2$ and $p=3$ ) with all (including off-diagonal) elements in the covariance matrix and with

$$
\begin{aligned}
g & =p^{2} \\
\rho_{j} & =1-\frac{2 p^{2}-1}{6 p n}\left(1+\frac{1}{j(j-1)}\right) \\
\omega_{2 j} & =-\frac{p^{2}}{4}\left(1-\frac{1}{\rho_{j}}\right)^{2}+\frac{p^{2}\left(p^{2}-1\right)}{24 n^{2} \rho_{j}^{2}}\left(1+\frac{2 j-1}{j^{2}(j-1)^{2}}\right)
\end{aligned}
$$

the probability of finding a smaller value of $-2 \rho_{j} \ln R_{j}$ is

$$
\begin{aligned}
& P\left\{-2 \rho_{j} \ln R_{j} \leq z\right\} \simeq P\left\{\chi^{2}(g) \leq z\right\}+ \\
& \omega_{2 j}\left[P\left\{\chi^{2}(g+4) \leq z\right\}-P\left\{\chi^{2}(g) \leq z\right\}\right],
\end{aligned}
$$

where $z=-2 \rho_{j} \ln r_{j}$ and $r_{j}$ is a particular realization (the observed value) of the stochastic variable $R_{j}$.

In the block-diagonal case, i.e., for azimuthal symmetry and diagonalonly data, the expression for $R_{j}$ is the same as here but the parameters in the distribution of $-2 \rho_{j} \ln R_{j}$ are different, see [34].

\subsection{The Loewner Order}

The above test statistics $Q$ and $R_{j}$ are all symmetric in the matrices $\boldsymbol{X}_{i}$ (and $\boldsymbol{X}_{j}$ ). This means that they are insensitive to the direction of change.

For scalar quantities it is easy to establish whether one quantity is larger than another, for example, we can check whether the difference between them is positive, negative or zero. For matrices this is a more complicated matter. The Loewner (or Löwner) order provides a partial ordering of matrices $[32,37,38]$. Here in our context it gives a direction of change: does the radar response $\boldsymbol{X}$ at time point one and $\boldsymbol{Y}$ at time point two increase or decrease (or does it possibly change structure or nature) between the two time points? To establish the Loewner order we calculate the definiteness of the difference $\boldsymbol{X}_{i}-$ $\boldsymbol{X}_{i+1}$. If $\boldsymbol{X}_{i}-\boldsymbol{X}_{i+1}$ is positive definite, we write $\boldsymbol{X}_{i+1}<_{L} \boldsymbol{X}_{i}$, if $\boldsymbol{X}_{i}-\boldsymbol{X}_{i+1}$ is negative definite, we write $\boldsymbol{X}_{i}<_{L} \boldsymbol{X}_{i+1}$.

\section{AN EXAMPLE, DISKO ISLAND, GREENLAND}

A test area near Qeqertarsuaq (Godhavn), Disko Island, Greenland provides opportunities for collecting SAR image data of buried test objects including humans hidden in snow caves, dog sleds and snow scooters (as well as test setups on the ice and snow cover of the Disko Island with tent and corner reflectors). Here, we work with two versions of the same data, one is single-look, another is multi-look (ENL = 3), both polarimetric F-SAR P-band (435 MHz) data near a (blue) cottage, see Figure 1. The data are acquired on 13 May 2015 (flightID 16) at three time points so $k$ above is 3

1. passID 05 starting at $16: 44: 53\left(t_{1}\right)$,

2. passID 06 starting at $16: 55: 43\left(t_{2}\right)$,

3. passID 07 starting at 17:07:00 $\left(t_{3}\right)$.

All three flights are with heading $175^{\circ}$. Flights are performed from a nominal altitude of 11,200 feet $(3,675 \mathrm{~m})$. The full polarimetry Pband sub-image data below are 450 rows by 3,000 columns. Pixels are $4.0 \mathrm{~m}$ (range) by $1.0 \mathrm{~m}$ (azimuth), images are $1.8 \mathrm{~km}$ by $3.0 \mathrm{~km}$. 


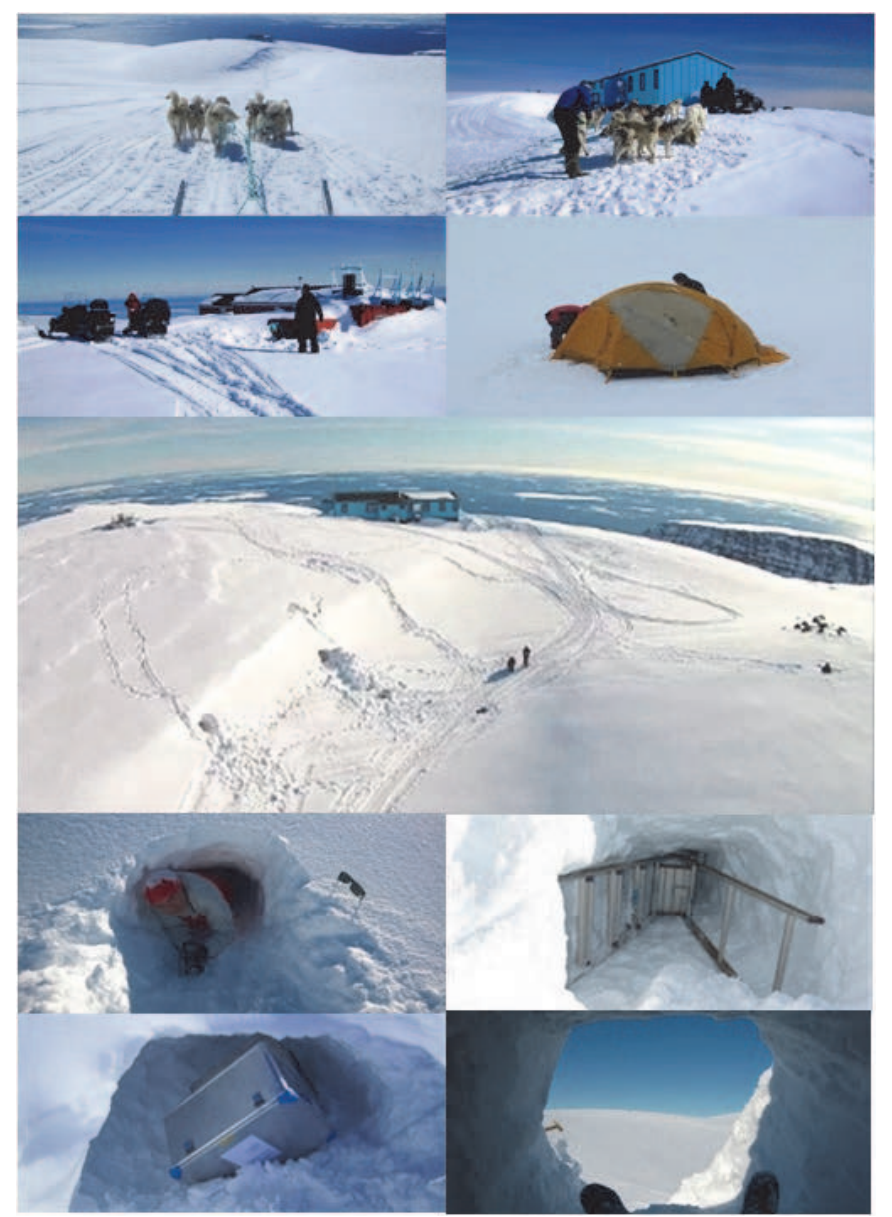

Fig. 1. Overview of experimentation area around the blue cottage, Disko Island, Greenland. Two snow scooters and two dog sleds were used as stationary and moving test objects during flights. Snow caves were dug for experiments with hidden objects: two persons, a ladder made of aluminium, and two aluminium boxes forming a dihedral corner structure with mostly double-bounce scattering. From [3].

\subsection{Multi-look data, $\mathrm{ENL}=3$}

Figure 2 shows $S_{h v} S_{h v}^{*}, S_{h h} S_{h h}^{*}$ and $S_{v v} S_{v v}^{*}$ as RGB for the three time points. Figure 3 shows change test statistics (top) $-2 \rho \ln Q$, (middle) $-2 \rho_{2} \ln R_{2}$ and (bottom) $-2 \rho_{3} \ln R_{3}$ for the same area as in Figure 2. Change at some time point occurs where values of $-2 \rho \ln Q$ are high (bright pixels), change between $t_{1}$ and $t_{2}$ occurs where values of $-2 \rho_{2} \ln R_{2}$ are high, change between $t_{2}$ and $t_{3}$ occurs where values of $-2 \rho_{3} \ln R_{3}$ are high. The horizontal stripes in Figure 3 indicating change just above the boxed zoom region are related to moving dog sleds and/or snow scooters. Their appearance in the images are shifted due to a velocity component perpendicular to the flight direction and smeared due to a component parallel to the flight direction.

Figure 4 shows a zoomed version of Figure 2 centered on the blue cottage seen in Figure 1. Figure 5 shows a zoomed version of Figure 3, same zoom as in Figure 4. Some of the detected targets in the zooms (right next to the digits 1 through 4) in Figure 5 top are objects buried in the snow, namely (here we focus on these objects)

1. a person,

2. an aluminium ladder,
3. two aluminium boxes,

4. another person.

We see that all four targets change between $t_{1}$ and $t_{2}$, and that only targets ' 1 ' and ' 4 ' change between $t_{2}$ and $t_{3}$. The Loewner order (not shown) can be used to illustrate whether targets come or go at different time points, see $[32,38]$. (The only target detectable at a $5 \%$ significance level is the very bright target below target ' 4 '; the remaining targets including the buried objects marked 1 through 4 exhibit less significant change.)

\subsection{Single-look data}

To improve the spatial resolution we work on single-look data also. The complex Wishart distribution based method in this case accommodates diagonal-only data but not polarimetric data. Figure 6 shows a zoomed version of the change test statistics in this case, same zoom as in Figure 5.

\section{CONCLUSIONS}

Using multi-look polarimetric covariance matrix data we detect the four buried objects albeit at low significance levels and judged visually with few false alarms.

Using single-look diagonal-only data causes buried object ' 4 ' to be detected at a higher significance level for $-2 \rho_{2} \ln R_{2}$ detecting change between $t_{1}$ and $t_{2}$. Contrary to this the multi-look change detected for object ' 4 ' by $-2 \rho_{3} \ln R_{3}$ between $t_{2}$ and $t_{3}$ is not detected by single-look diagonal-only data. Also, objects ' 1 ' and ' 3 ' are not detected. Further, because of the absence of multi-look speckle reduction, single-look detection gives more false positives and a less conspicuous spatial "togetherness" of the detected objects.

\section{REFERENCES}

[1] A. Reigber, R. Scheiber, M. Jäger, P. Prats-Iraola, I. Hajnsek, T. Jagdhuber, K. P. Papathanassiou, M. Nannini, E. Aguilera, S. Baumgartner, R. Horn, A. Nottensteiner, and A. Moreira, "Very-high resolution airborne synthetic aperture," in Radar Imaging: Signal Processing and Applications, Proceedings of the IEEE, 2013, vol. 101, pp. 759-783.

[2] K. Conradsen, A. A. Nielsen, and H. Skriver, "Determining the points of change in time series of polarimetric SAR data," IEEE Transactions on Geoscience and Remote Sensing, vol. 54, no. 5, pp. 3007-3024, May 2016, https://doi.org/10.1109/TGRS.2015.2510160.

[3] E. Krogager, "Multiband polarimetric SAR in Arctic scenarios," in International Conference on Radar, Brisbane, Queensland, Australia, 2018, https://radar2018.org/abstracts/pdf/abstract_ 171.pdf.

[4] R. Touzi, A. Lopes, and P. Bousquet, "A statistical and geometrical edge detector for SAR images," IEEE Transactions on Geoscience and Remote Sensing, vol. 26, pp. 764-773, 1988.

[5] E. J. Rignot and J. J. Van Zyl, "Change detection techniques for ERS-1 SAR data," IEEE Transactions on Geoscience and Remote Sensing, vol. 31, no. 4, pp. 896-906, 1993.

[6] Y. Bazi, L. Bruzzone, and F. Melgani, "An unsupervised approach based on the generalized Gaussian model to automatic change detection in multitemporal SAR images," IEEE Transactions on Geoscience and Remote Sensing, vol. 43, no. 4, pp. 874-887, 2005. 

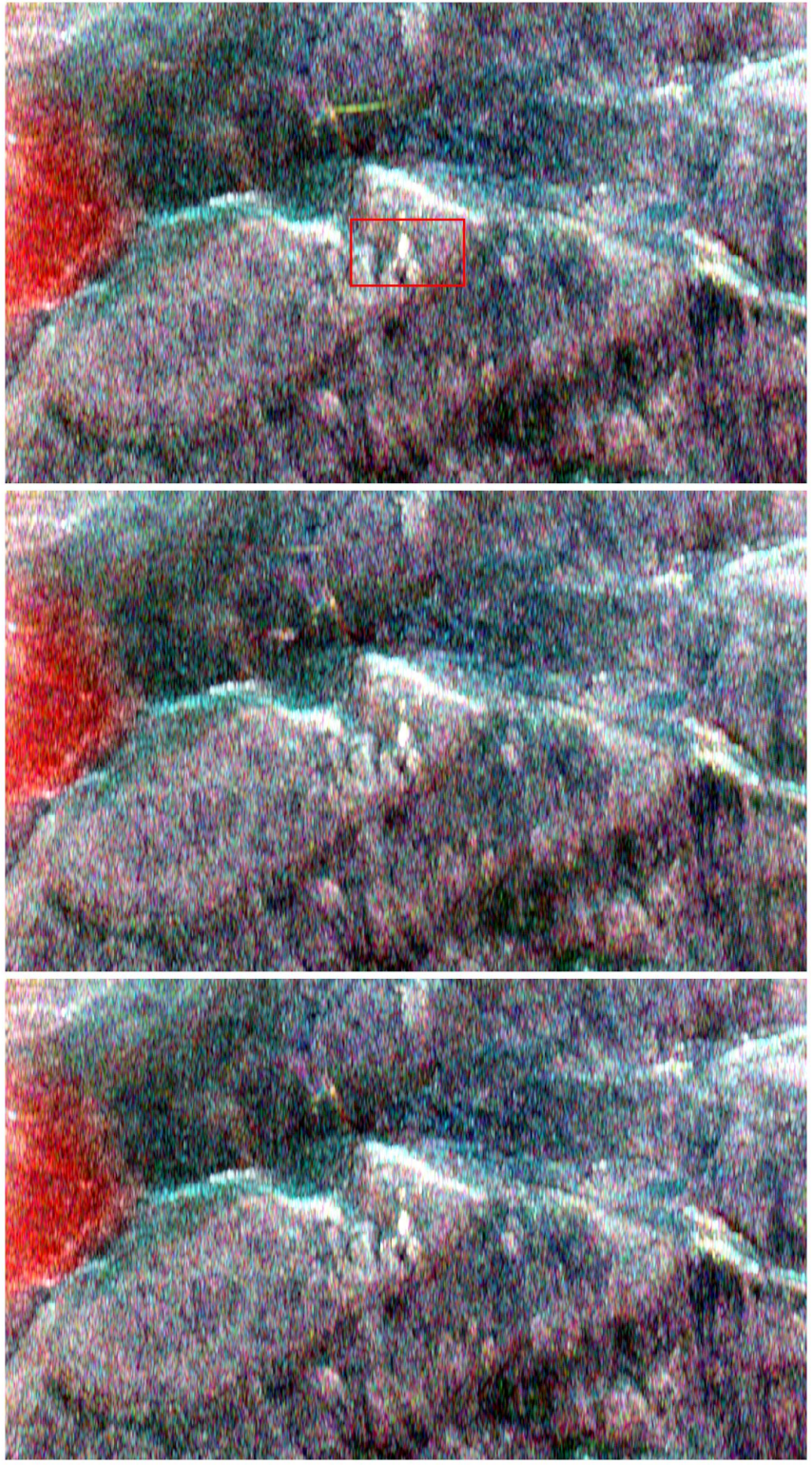

Fig. 2. Original data $S_{h v} S_{h v}^{*}, S_{h h} S_{h h}^{*}$ and $S_{v v} S_{v v}^{*}$ as RGB, three time points, note the red box on the top image $\left(t_{1}\right)$; this is the zoom area centered on the blue cottage.

[7] G. Moser and S. B. Serpico, "Generalized minimum-error thresholding for unsupervised change detection from SAR amplitude imagery," IEEE Transactions on Geoscience and Remote Sensing, vol. 44, no. 10, pp. 2972-2982, 2006.

[8] Y. Ban and O. A. Yousif, "Multitemporal spaceborne SAR data for urban change detection in China," IEEE Journal of Selected Topics in Applied Earth Observations and Remote Sensing, vol. 5, no. 4, pp. 1087-1094, 2012.

[9] H. Hu and Y. Ban, "Unsupervised change detection in mulitemporal SAR images over large urban areas," IEEE Journal of Selected Topics in Applied Earth Observations and Remote Sensing, vol. 7, no. 8, pp. 3248-3261, 2014.

[10] C. Carincotte, S. Derrode, and S. Bourennane, "Unsupervised
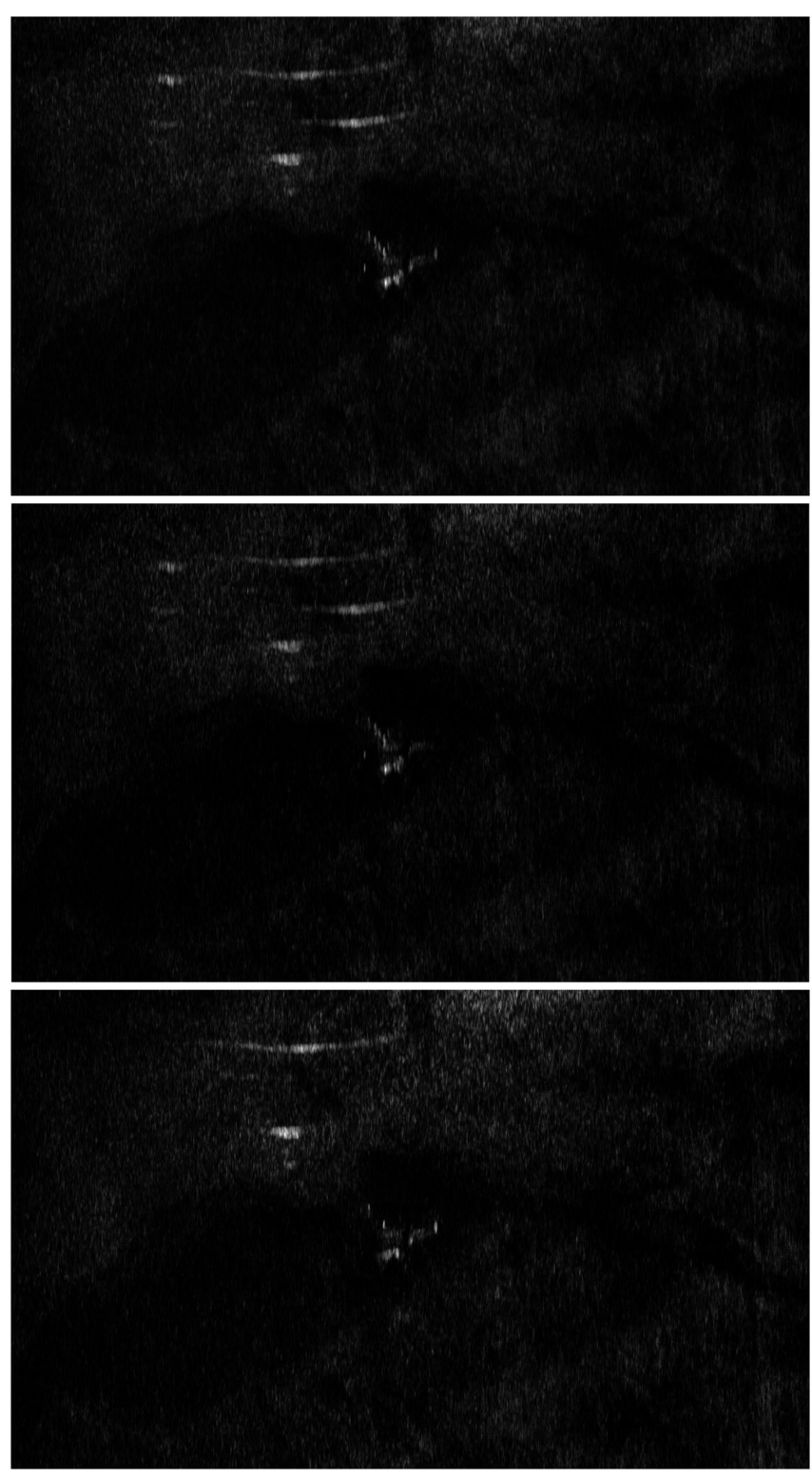

Fig. 3. Test statistics, $-2 \rho \ln Q$ (top), $-2 \rho_{2} \ln R_{2} \quad$ (middle), $-2 \rho_{3} \ln R_{3}$ (bottom). The (short) bright lines above the blue cottage are dog sled or snow scooter tracks.

change detection on SAR images using fuzzy hidden Markov chains," IEEE Transactions on Geoscience and Remote Sensing, vol. 44, no. 2, pp. 432-441, 2006.

[11] F. Bovolo and L. Bruzzone, "A detail-preserving scale-driven approach to change detection in multitemporal SAR images," IEEE Transactions on Geoscience and Remote Sensing, vol. 43, no. 12, pp. 2963-2972, 2005.

[12] T. Celik, "A Bayesian approach to unsupervised multiscale change detection in synthetic aperture radar images," Signal Processing, vol. 90, no. 5, pp. 1471-1485, 2010.

[13] M. Gong, Z. Zhou, and J. Ma, "Change detection in synthetic aperture radar images based on image fusion and fuzzy clustering," IEEE Transactions on Image Processing, vol. 21, no. 4, 


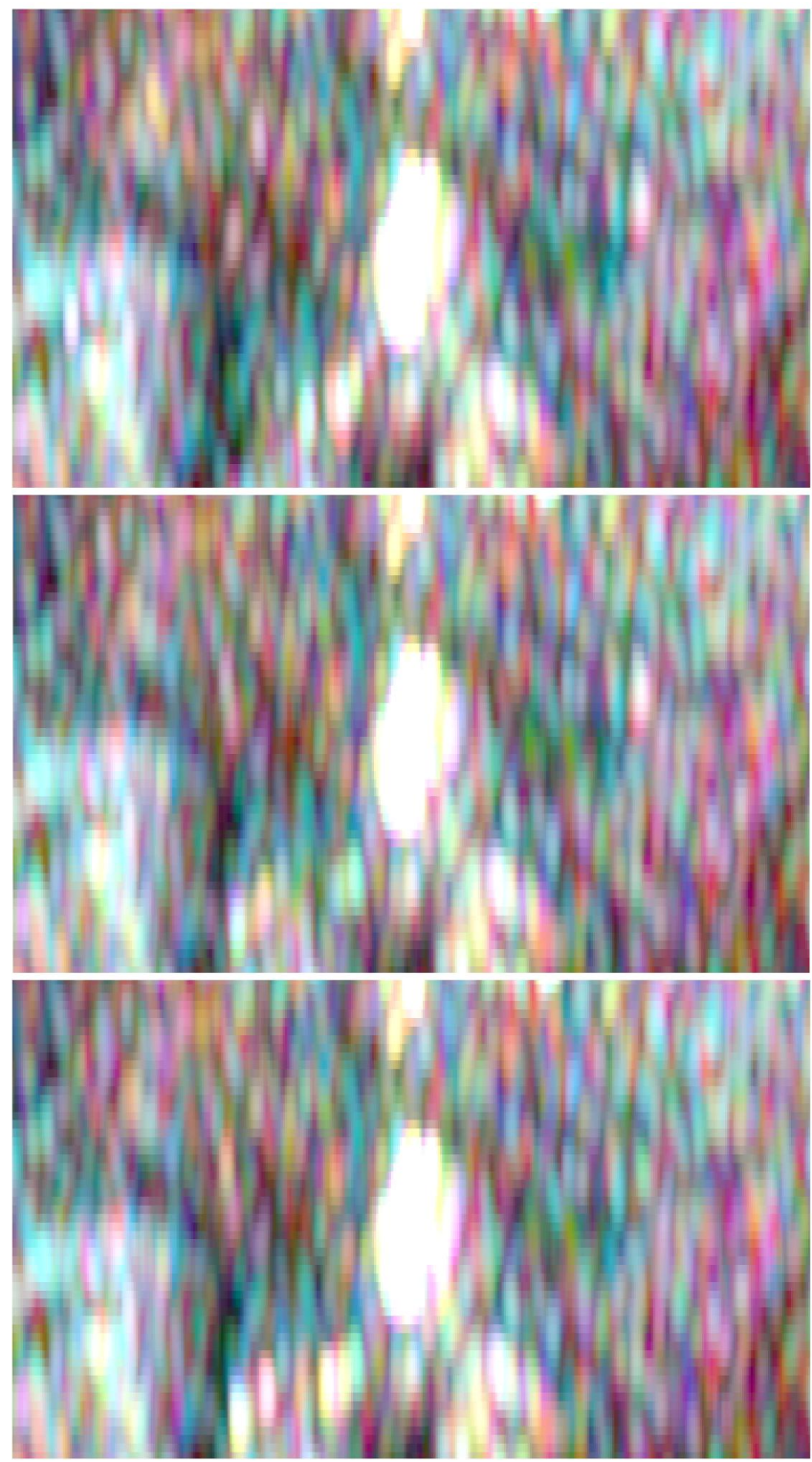

Fig. 4. Original data $S_{h v} S_{h v}^{*}, S_{h h} S_{h h}^{*}$ and $S_{v v} S_{v v}^{*}$ as RGB, three time points, zoom is centered on the blue cottage of images in Figure 2 .

pp. 2141-2151, 2012.

[14] F. Bovolo, C. Martin, and L. Bruzzone, "A hierarchical approach to change detection in very high resolution SAR images for surveillance applications," IEEE Transactions on Geoscience and Remote Sensing, vol. 51, no. 4, pp. 2042-2054, 2013.

[15] P. Gamba, F. Dell'Acqua, and G. Lisini, “Change detection of multitemporal SAR data in urban areas combining featurebased and pixel-based techniques," IEEE Transactions on Geoscience and Remote Sensing, vol. 44, no. 10, pp. 2820-2827, 2006.

[16] J. Inglada and G. Mercier, "A new statistical similarity measure for change detection in multitemporal SAR images and its extension to multiscale change analysis," IEEE Transactions on
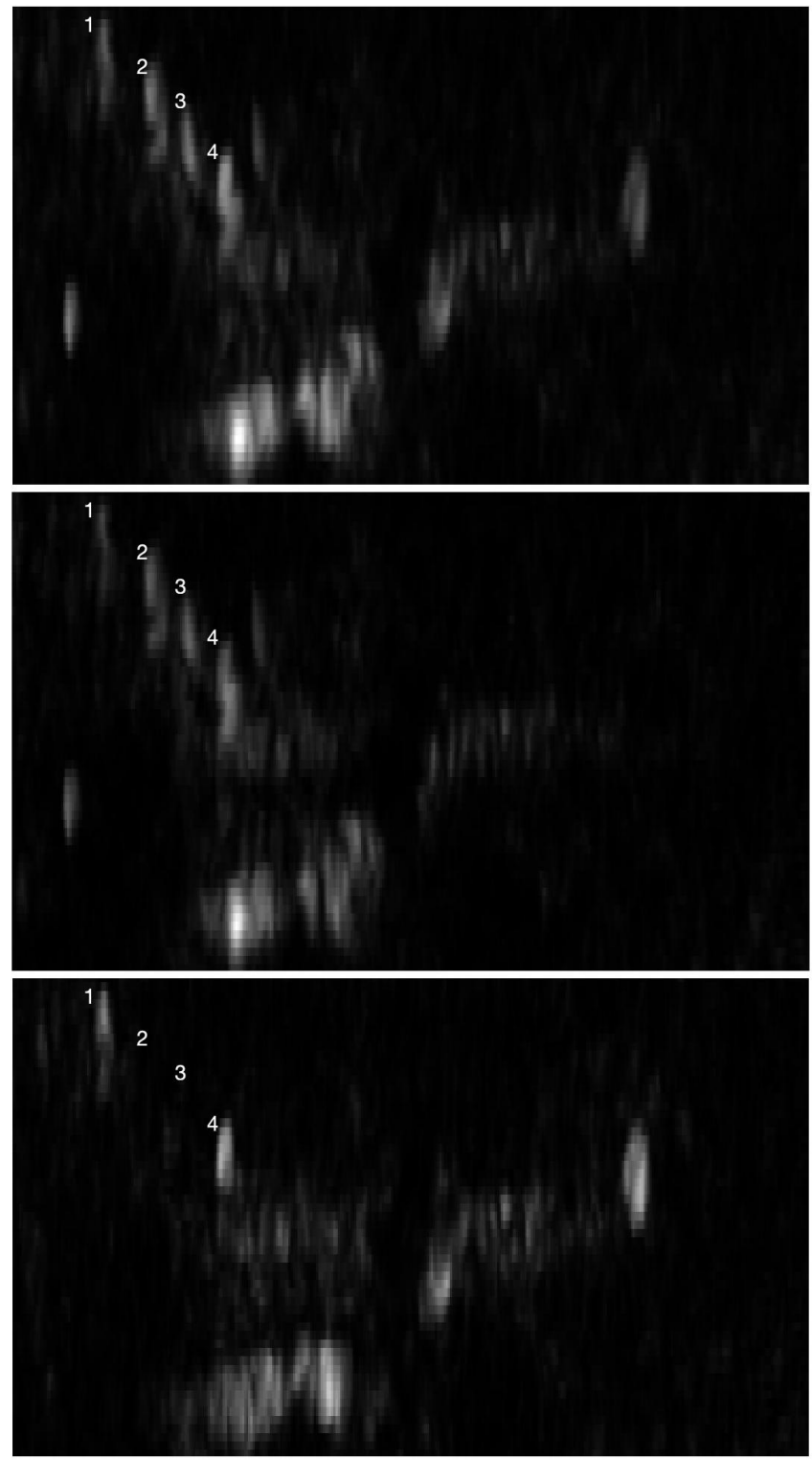

Fig. 5. Test statistics, same zooms on the blue cottage as in Figure 4; note labels ' 1 ', '2', ' 3 ' and ' 4 ' on the $-2 \rho \ln Q$ image. These labels mark the buried objects.

Geoscience and Remote Sensing, vol. 45, no. 5, pp. 1432-1445, 2007.

[17] F. Chatelain, J.-Y. Tourneret, and J. Inglada, "Change detection in multisensory SAR images using bivariate Gamma distributions," IEEE Transactions on Image Processing, vol. 17, no. 3, pp. 249-258, 2008.

[18] C. Pratola, F. Del Frate, G. Schiavon, and D. Solimini, "Toward fully automatic detection of changes in suburban areas from VHR SAR images by combining multiple neural-network modes," IEEE Transactions on Geoscience and Remote Sensing, vol. 51, no. 4, pp. 2055-2066, 2013.

[19] S. Hachicha and F. Chaabane, "On the SAR change detection review and optimal decision," International Journal of Remote Sensing, vol. 35, no. 5, pp. 1693-1714, 2014. 

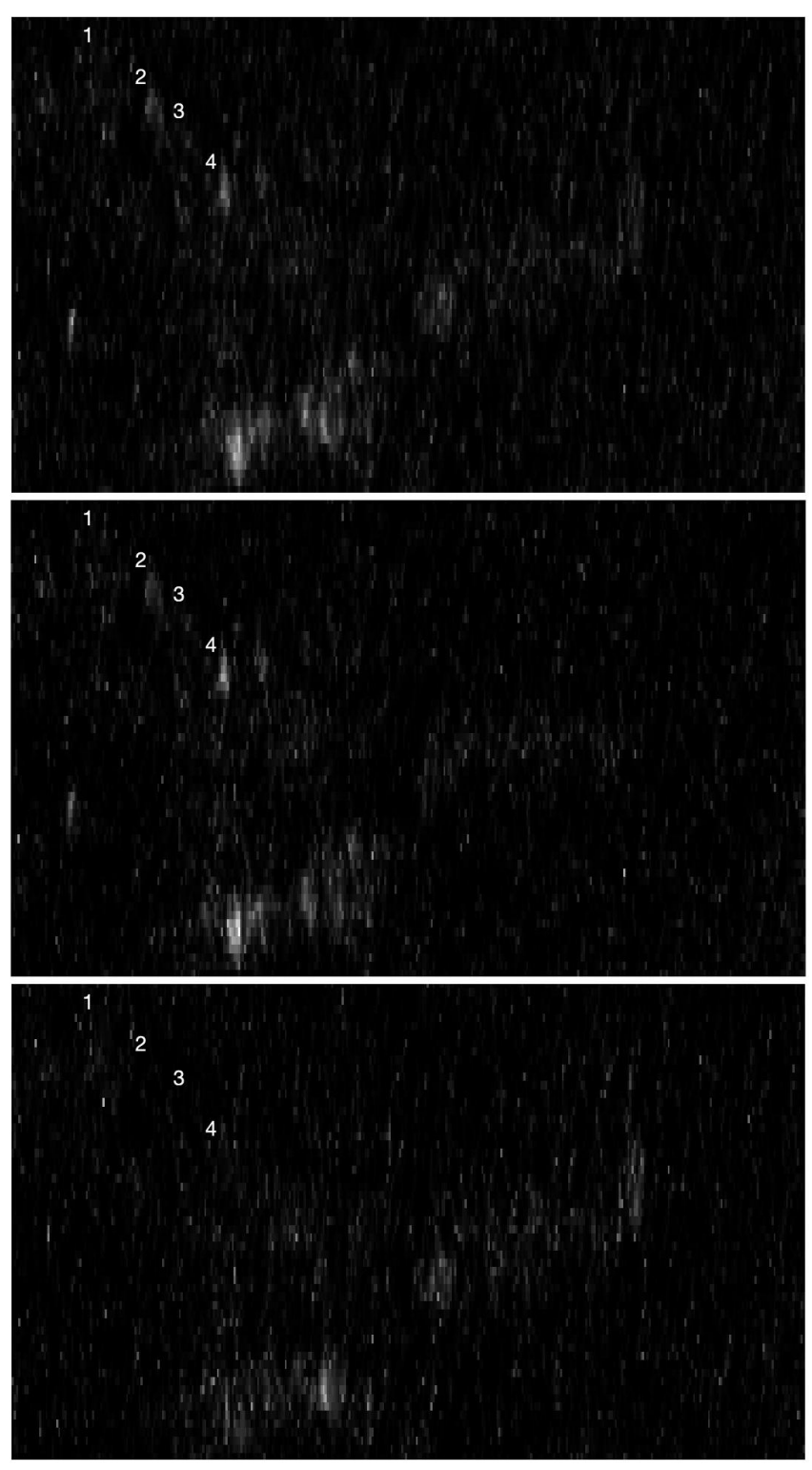

Fig. 6. Test statistics for single-look diagonal-only data, zoom.

[20] O. Yousif and Y. Ban, "Improving SAR-based urban change detection by combining MAP-MRF classifier and nonlocal means similarity weights," IEEE Journal of Selected Topics in Applied Earth Observations and Remote Sensing, vol. 7, no. 10, pp. 4288-4300, 2014.

[21] W. Dierking and H. Skriver, "Change detection for thematic mapping by means of airborne multitemporal polarimetric SAR imagery," IEEE Transactions on Geoscience and Remote Sensing, vol. 40, no. 3, pp. 618-636, Mar. 2002.

[22] M. Qong, "Polarization state conformation and its application to change detection in polarimetric SAR data," IEEE Geoscience and Remote Sensing Letters, vol. 1, no. 4, pp. 304-307, Oct. 2004.

[23] G. Moser, S. B. Serpico, and G. Vernazza, "Unsupervised change detection from multichannel SAR images," IEEE Geoscience and Remote Sensing Letters, vol. 4, no. 2, pp. 278-282,
Apr. 2007.

[24] G. Moser and S. B. Serpico, "Unsupervised change detection from multichannel SAR data by Markovian data fusion," IEEE Transactions on Geoscience and Remote Sensing, vol. 47, no. 7, pp. 2114-2128, Jul. 2009.

[25] K. Conradsen, A. A. Nielsen, J. Schou, and H. Skriver, "A test statistic in the complex Wishart distribution and its application to change detection in polarimetric SAR data," IEEE Transactions on Geoscience and Remote Sensing, vol. 41, no. 1, pp. 4-19, Jan. 2003, https://doi.org/10.1109/TGRS.2002.808066.

[26] P. Formont, F. Pascal, G. Vasile, J. P. Ovarlez, and L. FerroFamil, "Statistical classification for heterogeneous polarimetric SAR images," IEEE Journal of Selected Topics in Signal Processing, vol. 5, no. 3, pp. 567-576, Jun. 2011.

[27] A. Marino, S. Cloude, and J. Sanchez-Lopez, "A new polarimetric change detector in radar imagery," IEEE Transactions on Geoscience and Remote Sensing, vol. 51, no. 5, pp. 29863000, May 2013.

[28] V. Akbari, S. N. Anfinsen, A. P. Doulgeris, and T. Eltoft, "The Hotelling-Lawley trace statistic for change detection in polarimetric SAR data under the complex Wishart distribution," in IEEE IGARSS, Melbourne, Victoria, Australia, 21-26 July 2013, pp. 4162-4165.

[29] M. Liu, Hong Zhang, C. Wang, and F. Wu, "Change detection of multilook polarimetric SAR images using heterogeneous clutter models," IEEE Transactions on Geoscience and Remote Sensing, vol. 50, no. 7, pp. 7483-7494, Jul. 2014.

[30] W. T. Coombs, J. Algina, and D. O. Oltman, "Univariate and multivariate omnibus hypothesis tests selected to control type I error rates when population variances are not necessarily equal," Review of Educational Research, vol. 66, no. 2, pp. 137-179, 1996.

[31] A. A. Nielsen, K. Conradsen, H. Skriver, and M. J. Canty, "Visualization of and software for omnibus test based change detected in a time series of polarimetric SAR data," Canadian Journal of Remote Sensing, vol. 43, no. 6, pp. 582-592, 2017, https://doi.org/10.1080/07038992.2017.1394182.

[32] A. A. Nielsen, H. Skriver, and K. Conradsen, "The Loewner order and direction of detected change in Sentinel1 and Radarsat-2 data," IEEE Geoscience and Remote Sensing Letters, vol. 17, no. 2, pp. 242-246, Feb. 2020, https://doi.org/10.1109/LGRS.2019.2918636.

[33] J. J. van Zyl and F. T. Ulaby, "Scattering matrix representation for simple targets," in Radar Polarimetry for Geoscience Applications, F. T. Ulaby and C. Elachi, Eds. Artech, Norwood, MA, 1990.

[34] K. Conradsen, A. A. Nielsen, and H. Skriver, "Omnibus change detection in block diagonal covariance matrix polSAR data from Sentinel-1 and Radarsat-2," in prep., 2020.

[35] T. W. Anderson, An Introduction to Multivariate Statistical Analysis, John Wiley, New York, third edition, 2003.

[36] J. Schou, H. Skriver, A. A. Nielsen, and K. Conradsen, "CFAR edge detector for polarimetric SAR images," IEEE Transactions on Geoscience and Remote Sensing, vol. 41, no. 1, pp. 20-32, Jan. 2003, https://doi.org/10.1109/TGRS.2002.808063.

[37] S. K. Mitra, P. Bhimasankaram, and S. B. Malik, Matrix Partial Orders, Shorted Operators and Applications. Series in Algebra, vol. 10, World Scientific, Singapore, 2010.

[38] A. A. Nielsen, "Fast matrix based computation of eigenvalues and the Loewner order in polSAR data," IEEE Geoscience and Remote Sensing Letters (Early Access), Nov. 2019, https://doi.org/10.1109/LGRS.2019.2952202. 